MATEC Web of Conferences 22,01007 (2015)

DOI: $10.1051 /$ matec conf/20152201007

(C) Owned by the authors, published by EDP Sciences, 2015

\title{
Study on the Virtual Care System for the Elderly
}

\author{
Xuetao Hao*, Leilei Chen, Lisong Wei \& Jing Li \\ College of Communication Engineering, Xidian University, Xi'an, Shaanxi, China
}

\begin{abstract}
The virtual care system for the elderly is designed to make them healthier and safer under the increasingly serious aging situation. By using the image processing, speech recognition and relative sensors based on the intelligent terminals, the system accomplishes six functions: sports statistics, heart rate measurement drug manager, intelligent navigation home and reminder, SOS voice for help and kitchen reminder. Experiments show that it can provide health analysis and safe tutelage for the elderly comprehensively and accurately.
\end{abstract}

Keywords: the virtual care system; intelligent terminal; the elderly

\section{INTRODUCTION}

\subsection{Current aging population situation}

With the increasing number of aging population worldwide, the safety and health issues of the elderly have come to the stage as an urgent affair to be solved wisely and sensibly. In developed countries, especially in America and Japan ${ }^{[1]}$, numerous universities and research authorities have carried out researches supported by the government and society. The Medical Equipment Automation Research Center in University of Virginia conducted a study of smart home monitoring system in 2002 with inexpensive sensors to track activities of the elderly ${ }^{[2]}$. The real-time monitoring can be realized through the analysis of regular patterns in data mining techniques. The implementation of unsupervised health surveillance was proposed by Japan's National Institute for Longevity Department of Gerontology. Moreover, the system architecture analysis laboratory in France is conducting remote monitoring system with multi-sensors for the elderly living alone ${ }^{[2]}$.

\subsection{Pertinent research}

At present, domestic research on elderly health care area is relatively less than that of abroad ${ }^{[3]}$. Professor Anyu Chen created a behavior analysis and abnormal alarm system for the elderly living alone, which was a tremendous attempt to attend them with initiative judgment to the conditions of the elderly based on the detected signals. Yet, the system intelligence property is low due to the appliance of SCM. Image Analysis and Machine Intelligence Laboratory of Shanghai Jiao Tong University proposed and implemented home remote medical monitoring and counseling intelligent alarm system with certain intelligence. However, due to the home health monitors and controllable "Wanxiangyun" equipment with fairly high cost, it is not suitable for extensive promotion. Nowadays, some manufacturers have introduced varieties of systems for home care towards the elderly. But most of them are constrained to the high cost, small scale and low portability, which lead consumers to a dreadful experience. Thus, observing the increasing penetration and influence of intelligent terminals, we desire to utilize them to develop the virtual care system, which can truly manage the health and safety issues of elderly with great promotion value.

\section{SYSTEM PROGRAMMING}

\subsection{System structure design}

System design focuses on two modules: health analysis and safety tutelage. The weaknesses such as low portability and high cost appeared in present techniques have been surmounted with information collection through Gravity Sensor, camera, Acceleration Sensor, GPS and handset (see Figure 1) in intelligent mobile terminals, which characterizes the system with high portability, low cost and non-peripherals.

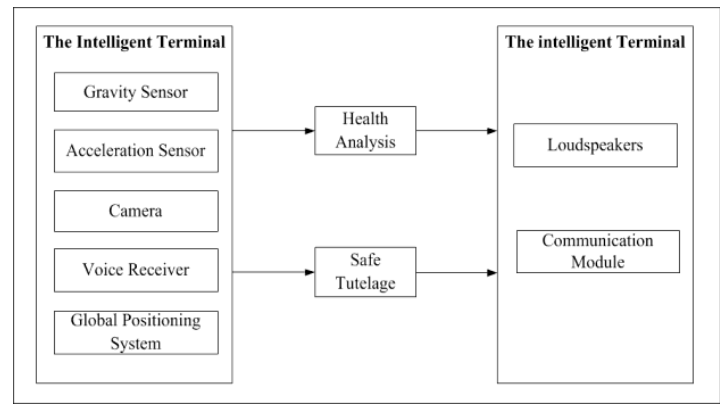

Figure 1. System structure design

\subsection{System frame design}

The system frames and function modules are designed based on the demands of the elderly (see Figure 2). It

*Corresponding author: joker abc@163.com

This is an Open Access article distributed under the terms of the Creative Commons Attribution License 4.0, which permits unrestricted use, distribution, and reproduction in any medium, provided the original work is properly cited. 
exploits mobile intelligent terminals combined with related sensors, image processing and speech recognition technology to achieve the six functions for the health care managements and security tasks.

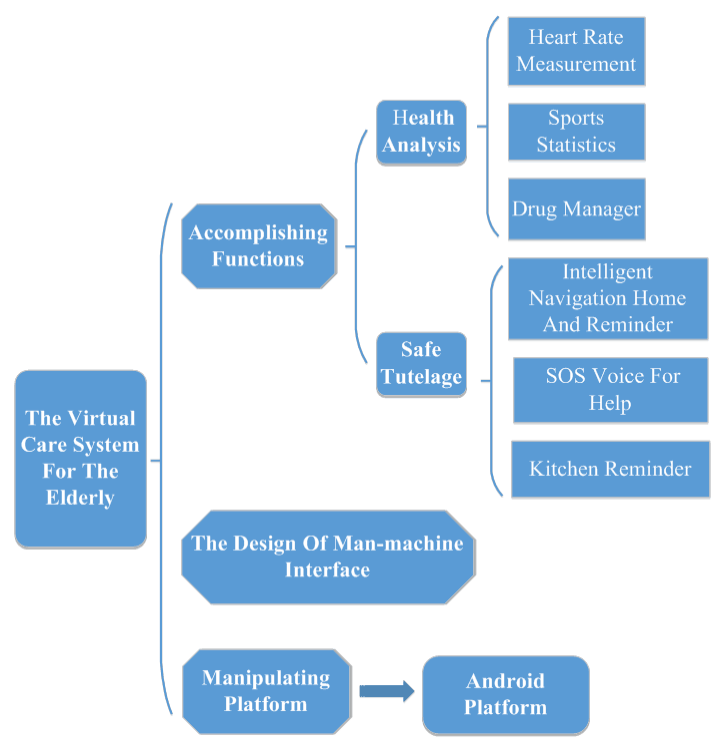

Figure 2. System frame

\section{SYSTEM INTERFACE DESIGN}

Considering that users are mainly composed of elderly, we design the interface based on the principle: concise, clear and strong resolution. Thus, we implement flat interface with high-contrast and vivid pictures which contribute to the understanding of the elderly towards the application (see Figure 3).

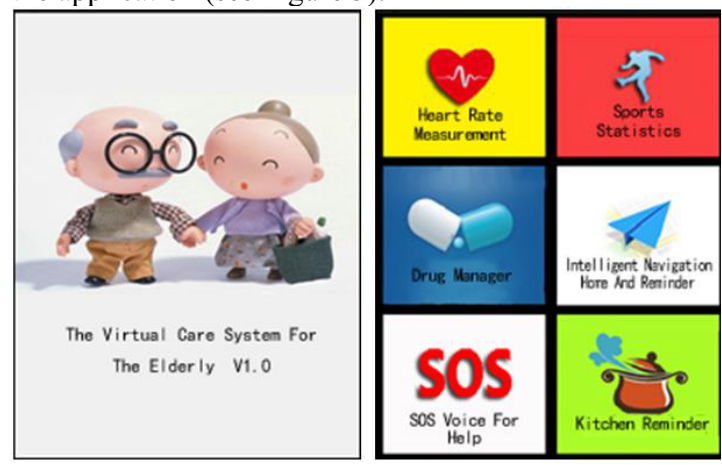

Figure 3. User interface

\section{SYSTEM FUNCTION MODULE DESIGN}

\subsection{Heart rate measurement}

The elderly can know their own heart rate whenever and wherever they are through their cell phones with- out any other peripherals. Every time after the measurement, the system will send a feedback to the user with certain advices towards this mensuration (shown in Figure 4). The heart rate monitoring implements image processing techniques.

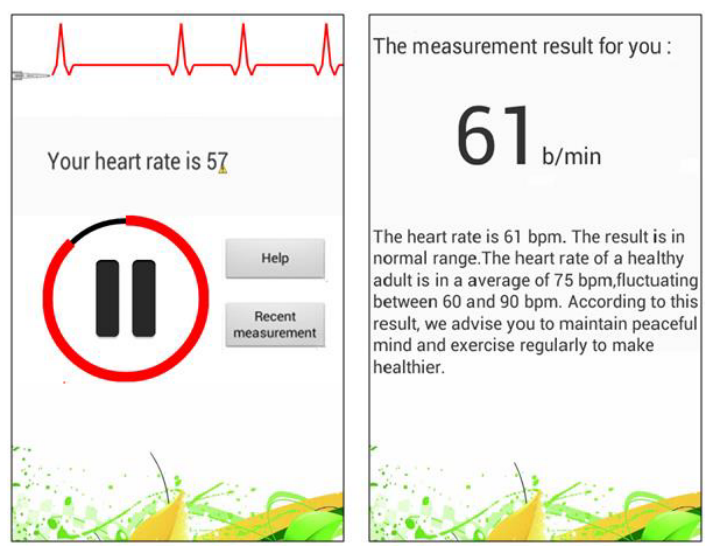

Figure 4. Heart rate measurement \& feedbacks

The heart rate measurement and monitoring algorithm are formulated on the basis of the theory:

The color of the capillaries in the finger will change cyclically in brightness with pulse beats, and correspondingly, the component R captured in RGB (red, green and blue) images will exhibit cyclical fluctuations ${ }^{[4]}$.

The brightness deviation YUV image taken by the cameras is shown in Figure 5. It is obvious that the brightness varies dramatically in different positions. Thus, we transform the YUV image into RGB image in formulas (1):

$$
\begin{aligned}
& R=Y+1.4075 \times(\mathrm{V}-128) \\
& \mathrm{G}=Y-0.3455 \times(\mathrm{U}-128)-0.7169 \times(\mathrm{V}-128) \\
& \mathrm{B}=\mathrm{Y}+1.779 \times(\mathrm{U}-128)
\end{aligned}
$$

Where R, G, B respectively represents red, green and blue component value in each pixel; $\mathrm{Y}$ represents brightness degree; $\mathrm{V}$ represents aberration between $\mathrm{R}$ and $\mathrm{Y}$; $\mathrm{U}$ represents the aberration between $\mathrm{B}$ and $\mathrm{Y}$.

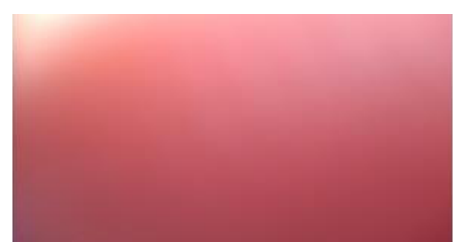

Figure 5. YUV image

Then we establish the statistics of component $\mathrm{R}$ in RGB image and suppose $\mathrm{R}$ to be $\mathrm{X}^{[5]}$. The present red pigment density will be concluded and be put into the 
array of $\mathrm{R}, \mathrm{X}$ and $\mathrm{S}$ (the area of finger which is taken in photos):

$$
\rho_{\mathrm{R}}=\frac{\mathrm{X}}{\mathrm{S}}
$$

Taking all the red pigment density of system array into consideration, we can obtain the mean as follows:

$$
\overline{\rho_{\mathrm{R}}}=\frac{\sum_{1}^{\mathrm{n}} \rho_{\mathrm{Rn}}}{\mathrm{n}}
$$

Where $\mathrm{n}=1,2,3 \ldots$ represents the times of measuring while $\rho_{\mathrm{Rn}}$ represents the value of red pigment density at the $\mathrm{n}^{\text {th }}$ time. The system will record the beats $m$ automatically from the start to the destination based on the prerequisite: it will be measured as a heartbeat when $\rho_{R}<\overline{\rho_{R}}{ }^{[6]}$. Thus, we can obtain the heart rate $\mathrm{W}:$

$$
\mathrm{W}=\frac{\mathrm{m}}{\mathrm{t}-\mathrm{t}_{0}}
$$

Where, $t$ represents the time of ending measurement, while $t_{0}$ represents the start time.

\subsubsection{Functional testing}

We test the accuracy of heart rate monitoring in comparison with the app named "heart rate", which possesses the highest download amount in Android Software Market.

- Set the experimental group

Using the heart rate measure module, we design to do the one-minute test towards 50 randomly selected users and record the consequences.

- Set the contrast group

Using the heart rate measure module, we load in the app market to do the one-minute test towards those 50 users in the experimental group and then record the consequences.

\section{- Statistics}

We find the average error rate is less than $10 \%$, meaning a great consequence that can reflect the heart beat condition decently (see Figure 6).

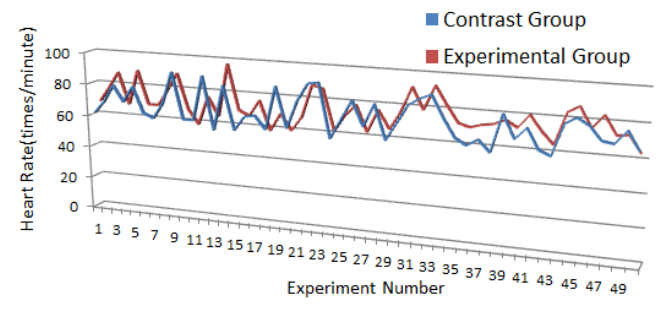

Figure 6. Experiment data analysis

\subsection{Drug manager}

This function module aims to remind the elderly of taking medicine on schedule for the common oblivion phenomenon ${ }^{[7]}$. Users can input the drug name, period time and dose based on their own will (shown in Figure 7). Accordingly, the module will manage the schedule and give users reminders through system clock. Therefore, there will be no more over dose and the elderly will get the drug information precisely and minutely. The adjustment can be conducted based on personal situation of the elderly when clicking the drug name in the interface as shown in Figure 7.
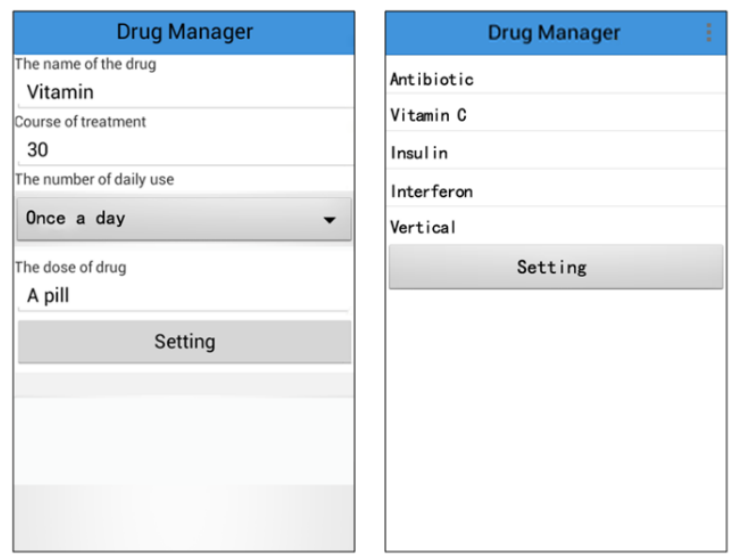

Figure 7. Drug setting and interface

\subsection{Sports statistics}

The function module is designed to count the exercise and offer a more objective and precise data analytics for the demand of the elderly to preserve their health. The module implements the theory: The center of gravity will move up and down when you are walking [8]. The sports statistics are counted through the data collection and Gravity Sensor exploitation (see Figure 8).

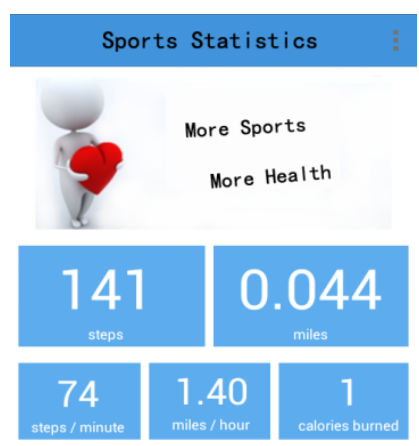


Figure 8. Sports statistics interface

The variance of acceleration can be calculated through the measurement of acceleration components in three dimensions with the Gravity Sensor ${ }^{[9]}$. We can obtain as follows:

$$
S=\left|V_{t}-V_{t-1}\right|
$$

Where $S$ represents the variable value of acceleration; $V_{\mathrm{t}}$ represents the average acceleration value in 3 dimensional directions; $V_{\mathrm{t}-1}$ records the average acceleration value at the moment of $\mathrm{t}-1$.

The sensitivity and step length can be set in the menu. On the conditions that the variance of acceleration $\mathrm{R}$ is greater than the value of sensitivity, it can be judged that the user has walked a step further. Recording the statistics of steps, we can get the distance and realize the sports statistics.

\subsubsection{Functional testing}

We convene 50 elderly to experience the function module and get the statistics compared with the realistic step amount. The error rate is controlled reasonably within $18 \%$, thus it is proved that the sports statistics are relatively precise.

\subsection{SOS voice for help}

There will be a quantity of emergency situation that the elderly need help promptly. The function modules simplify the process of editing and sending SMS. What elderly required to do is just to speak "help" to the phone, and the message will be sent to the pre-set emergency contacts automatically.

When the module is turned on (see Figure 9), it will capture the voice information periodically in the background based on iFLYTEK (a voice recognition module).

On capturing information, the module will transform the audio signals into string information ${ }^{[10]}$. If the string the module captured corresponds with the string of "help", message will be sent immediately.
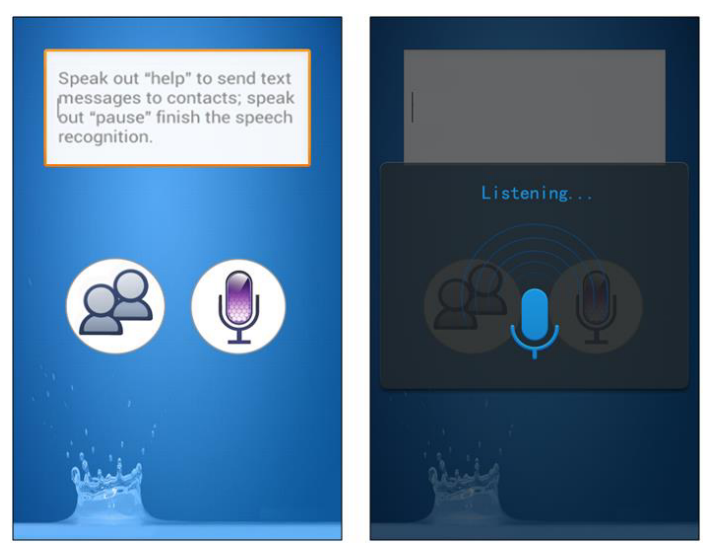

Figure 9. User interface

\subsubsection{Functional innovation}

The module can be implemented without connecting with internet, which overcomes the issue of not matching when lacking internet.

The recognition library is updating through experiments, which means the module will record the similar sounding words while being tested and plug them into the data base. Therefore, with the escalating amount of test, the recognition rate will improve itself.

\subsubsection{Functional testing}

- Set the experimental group

Choose 50 users randomly and let them speak "help" at a distance of 1 meter away from the cell phone. Every user should speak "help" ten times at least. Record the data and analyze the recognition rate.

- Statistics

We find the average recognition rate is approximately $67 \%$, which means the function module is decent and reliable to offer assistance when the elderly are in trouble.

\subsection{Kitchen reminder}

The elderly living along sometimes will forget the stuffs that need to be dealt with, which leads to a chain of danger. Thus, this function module is established to assure safety of the elderly in the kitchen through 4 aspects: boiling, stewing, steaming and making soup. The four different alarms for those four events are respectively recorded in prerequisite to remind them of dealing with different kitchen events (see Figure $10)$.

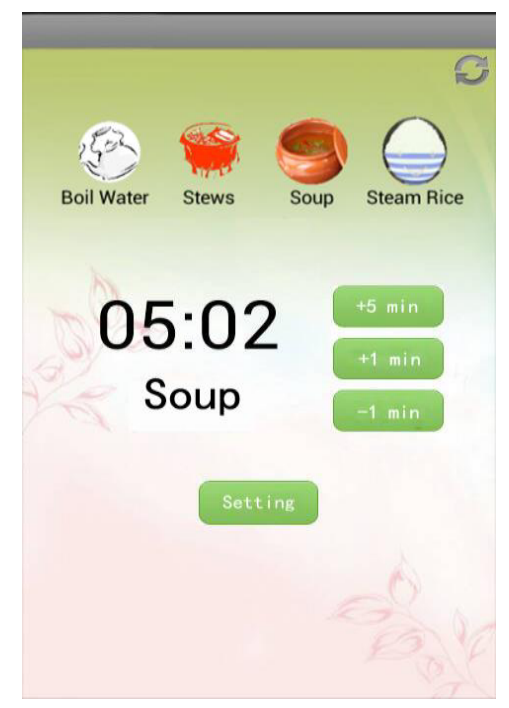

Figure 10. Kitchen reminder 


\subsection{Intelligent navigation home and reminder}

It is common that relatives are always apprehensive for the elderly's safety especially under the situation that they have to go out for some affairs. The function is designed to solve the issue by informing the pre-set contacts when the elderly exceed the reasonable range. Meanwhile, the reminders will be offered through pulling instructions in cellphones.

GPS (Global Positioning System) is implemented by the system to auto-record the route and location of the elderly (shown in Figure 11). On inputting the range in the setting function (shown in Figure 12), system will remind the elderly that they have passed the area of pre-set and ask for the permission to navigate home. If users approve it and click "yes", the minimum distance route will be programmed automatically as shown in Figure 11. When the user is out of the range for a relatively long period, relatives will be informed through texts with GSM module ${ }^{[11]}$. Also, the general location will be provided to find the users.

The coordinates of longitude and altitude are obtained through utilizing GPS, which are implemented to get the deviation from the present pot to the safety location on the basis of pre-set residential address through the method of counting the departure distance.
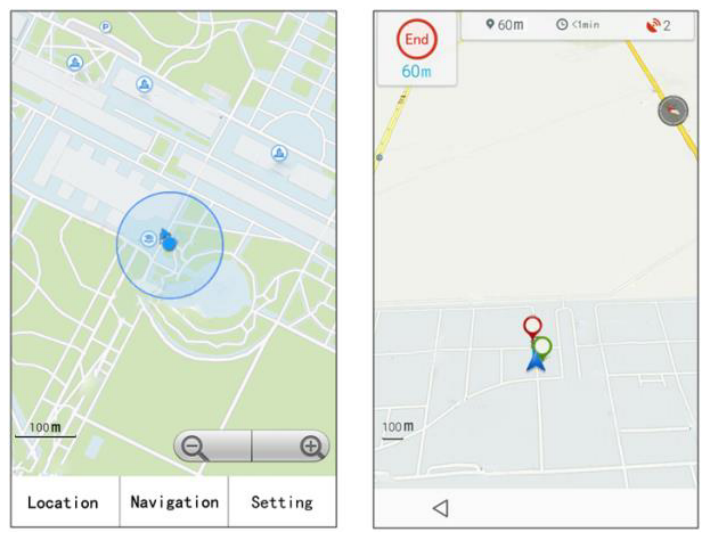

Figure 11. Locating \& Navigating

The algorithm implemented is shown as follows:

1) Calculate the arc length from the present position coordinates to the center in the circle of longitude:

$$
L=\left|X_{1}-X_{2}\right| \times \pi \times \mathrm{R} \times \cos \left(Y_{1}\right) / 180
$$

Where $\mathrm{L}$ represents the arc length from the present position coordinates to the center; $X_{1}, X_{2}$ respectively represents the central longitude coordinate and the present position longitude coordinate; $\mathrm{R}$ represents the radius of Earth and $Y_{1}$ represents the central position latitude coordinate.

2) Calculate the arc length from the present position coordinates to the center in the circle of latitude:

$$
S=\left|Y_{1}-Y_{2}\right| \times \pi \times \mathrm{R} / 180
$$

Where $S$ represents the arc length from the present position coordinates to the center in the circle of latitude; $Y_{1}$ represents the central position latitude coordinate and $Y_{2}$ represents the present position latitude coordinate.

3) Calculate the deviation distance:

$$
D=\sqrt{L^{2}+\mathrm{S}^{2}}
$$

Where D represents the distance from the present position coordinate to the central spot, namely, the deviation distance.

When the elderly click "Open the position dynamic monitor" (see Figure 11), the distance variance will be figured up through the algorithm. Thus, the safety is protected by the function module through reminders both to the users and pre-deserved emergency contacts.

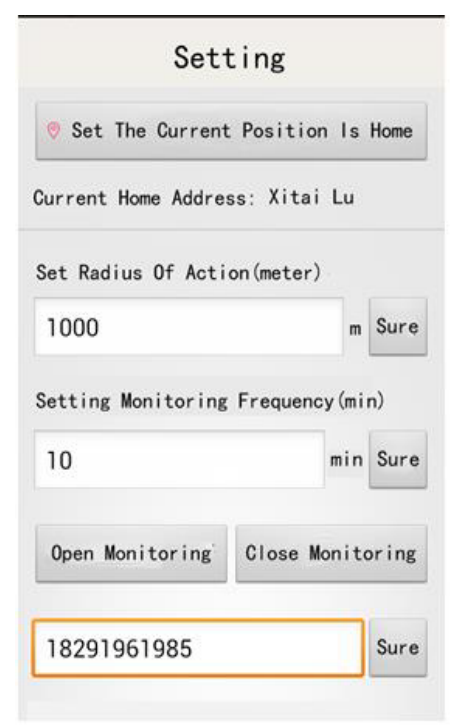

Figure 12. Setting

\section{CONCLUSION}

This paper proposes a method which takes advantages of intelligent cell phone terminals combined with relative sensors, image processing and voice recognition to realize the virtual care system for the elderly.

The system can do real-time monitoring and analyze the health and safety condition comprehensively, which assures the safety of elderly. Also, the non-periphery concept is conducted with relatively low cost and high portability, which indicates its great promotion value. 


\section{ACKNOWLEDGEMENT}

This paper is sponsored by National Undergraduate Training Programs for Innovation and Entrepreneurship (GN: 201410701003).

\section{REFERENCES}

[1] Ma Baoqing, Tang Yiping, Yan Hangchen. 2013. Monitoring system for elderly people living alone based on omni-directional vision, Computer Engineering, 39(8).

[2] Tang Yiping, Gu Xiaokai, Sun Hongjie, et al. 2007. Elder health status monitoring through analysis of activity. Computer Engineering and Applications, 43(3).

[3] Chen Dirong. 2012. Design of a system circuit for intelligent Terminal nursing home applications, Journal of Hangzhou Dianzi University.

[4] Tingting Bi. 2013. The research of ECG analysis system based on Android, Journal of Nanjing University of Posts and Telecommunications.

[5] Tang Hongling. 2013. The design and implementation of android heart rate monitoring application based on signal process, Journal of Northeastern University.

[6] Qi Jiaxue, Zhao Peng, Jiao Teng, Li Bin, Xu Xinping, Luo Jia, Wang Nan. 2006. Design of Atmega64L-based MCU in heart-rate monitoring system, Chinese Medical Equipment Journal.

[7] Bi Hanfeng. 2012. Hospital medicine management information system design and implementation, China Academic Journal Electronic Publishing House.

[8] Shi Xindong, Cheng Yang, Zhang Yu, Zhang Xun. 2011. Research on intelligent fitness partner based on the acceleration and photoelectric sensors, Chinese Journal of Medical Instrumentation, 35(5).

[9] Han Wenzheng, Feng Di, Li Peng, Ma Wenchao. 2012. Design of pedometer based on acceleration sensor LIS3DH, Transducer and Microsystem Technologies, 31(11).

[10]Liu Xiantao. 2013. Design and implementation of speech recognition client system based on android platform, China Academic Journal Electronic Publishing House.

[11]Xie Xueting. 2013. Software design based on GPS/GSM positioning monitoring system for the elderly and children, Journal of Suzhou University. 